ANNALES

POLONICI MATHEMATICI

XV (1964)

\title{
Une variante de la méthode de $M$. Leja pour l'approximation de la représentation conforme
}

\author{
par W. KLEINER (Kraków)
}

1. Préllminaires. Soit $D$ un domaine plan contenant le point à l'infini, dont la frontière $C$ est une courbe simple de courbure bornée et de longueur $|C|<2$. Nous emploierons la représentation paramétrique naturelle de $C: z=z(s),\left|z^{\prime}(s)\right|=1, z(s+|C|)=z(s)$. Pour une mesure (signée) [1] $\mu$ sur $C$ le potentiel est défini par

$$
U^{\mu}(z)=\int \log |z-\zeta|^{-1} d \mu(\zeta),
$$

l'énergie mutuelle de $\mu_{1}$ et $\mu_{2}$ par

$$
\left(\mu_{1}, \mu_{2}\right)=\int U^{\mu_{1}} d \mu_{2},
$$

et $\|\mu\|^{2}=(\mu, \mu)$ est appelé énergie de $\mu$; elle est positive sauf pour $\mu \equiv 0$. L'ensemble des mesures d'énergie finie est un espace préhilbertien pour le produit scalaire (2).

2. La mesure d'équilibre. Parmi les mesures $\mu$ sur $C$ pour lesquels $\mu(C)=1$ il existe [3] une mesure $\eta$ d'énergie minime; elle est appelée mesure d'équilibre sur $C$.

Le diamètre transfini [2], [10]

$$
d=d(C)=\exp -\|\eta\|^{2} .
$$

La fonction $w=f(z), f(\infty)=\infty, f^{\prime}(\infty)=1$, qui donne la représentation conforme de $D$ sur le cercle $|w|>d$ est liée à $\eta$ par les relations

$$
\left|\frac{d f}{d z}\right|=2 \pi \eta^{\prime}=2 \pi \frac{d \eta}{d s} \operatorname{sur} C, \quad \log \frac{1}{f(z)}=\int \log \frac{1}{z-\zeta} d \eta(\zeta)
$$

les branches des logarithmes étant convenablement choisies.

Par le principe de variation de Lindelöf [7] on a pour tout $z_{0} \in C$, $\left|f^{\prime}\left(z_{0}\right)\right| \leqslant\left|g^{\prime}\left(z_{0}\right)\right|=\varkappa d$, où $\varkappa$ est la borne supérieure de la courbure de $C$ et $g$ donne la représentation conforme de l'extérieur du cercle de rayon $1 / x$ tangent intérieurement à $C$ au point $z_{0}$ sur $|w|>d$, de manière que $g(\infty)=\infty$ 
3. Mesures discrètes. Soit $\varepsilon(z)$ la mesure portant la masse unité au point $z$, c'est-à-dire $\int h(\zeta) d \varepsilon(z)=h(z)$ pour toute $h$ continue. Toute mesure de la forme

$$
\tau=\sum_{i=1}^{n} m_{i} \varepsilon\left(z_{i}\right) \quad\left(z_{i} \epsilon C\right)
$$

est appelée mesure discrète sur $C$. Nous convenons une fois pour toutes que $m_{i} \geqslant 0, \sum_{i=1}^{n} m_{i}=1$. Posons par définition

$$
\begin{gathered}
\|\tau\|_{0}^{2} \stackrel{\text { df }}{=} \int_{z \neq \zeta} \log |z-\zeta|^{-1} d \tau d \tau=\sum_{i \neq k} m_{i} m_{k} \log |z-\zeta|^{-1}, \\
\|\tau\|_{n}^{2}=\int U_{n}^{\tau}(z) d \tau, \quad U_{n}^{\tau}(z)=\int \log _{n}|z-\zeta|^{-1} d \tau, \\
\log _{n} t=\min \{\log t, \log n / \vartheta\}
\end{gathered}
$$

où $\vartheta>0$ est un nombre fixe quelconque, que nous allons préciser au $\mathrm{n}^{0} 5$.

4. La méthode de M. Leja. Pour un $n$ quelconque fixons dans (5) $m_{i}=1 / n(i=1, \ldots, n)$ et cherchons les points $z_{i}=\eta_{i n}$ tels que $\|\tau\|_{0}^{2}$ soit la plus petite possible. Soit $\eta_{n}=\sum n^{-1} \varepsilon\left(\eta_{i n}\right)$ la mesure obtenue. M. Leja a démontré [8], [9] que

(8) $f_{n}(z) \stackrel{d \underline{I}}{=}\left[\left(z-\eta_{1 n}\right) \ldots\left(z-\eta_{n n}\right)\right]^{1 / n}=\exp -\int \log (z-\zeta)^{-1} d \eta_{n} \rightarrow f(z) \quad(z \in D)$.

Nous avons donné une estimation de cette convergence dans [4].

La détermination numérique des points extrémaux $\eta_{i n}$ est un problème d'analyse élémentaire, qui semble bien résoluble à l'aide d'un calculateur électronique. On peut néanmoins tenter de réduire le temps exigé pour le calcul et de simplifier le programme.

Nous proposons ici une variante de cette méthode, où l'on opère pareillement avec des mesures discrètes portées par $n$ points, mais où ces points sont fixés et les masses sont à choisir de manière que (16) soit minimalisée. C'est alors une forme quadratique à coefficients donnés dont on cherche le minimum. Le programme pour un calculateur est extrêmement simple et une machine à paramètres techniques très modestes peut fournir la solution en peu de temps.

5. Partageons $C$ en $n$ ares $C_{i n}$ de longueurs peu différentes et soit $z_{\text {in }}$ le centre de $C_{i n}$ par rapport à la longueur de l'are sur $C$. Plus précisément, nous supposons que

$$
\vartheta / n \leqslant\left|z_{i n}-z_{k n}\right|(i \neq k) \quad \text { et } \quad\left|C_{i n}\right| \leqslant \theta / n(i, k=1, \ldots, n),
$$


où les nombres positifs $\vartheta, \theta$ ne dépendent pas de $n$ (la différence $\theta-\vartheta$ a une influence, bien que faible, sur les erreurs des approximations ci-dessous).

Soit $K_{n}$ la classe des mesures discrètes sur $z_{i n}$ de ,densité bornée":

$$
K_{n}=\left\{\tau: \tau=\sum_{i=1}^{n} m_{i} \varepsilon\left(z_{i n}\right), 0 \leqslant m_{i} \leqslant x d \cdot \theta / n, \sum_{i=1}^{n} m_{i}=1\right\} .
$$

Nous entendons dorénavant, dans (7), par $\vartheta$ le même nombre que dans (9).

Il existe une $\tau_{n} \in K_{n}$ minimale pour \|\|$_{n}^{2}$, c'est-à-dire $\left\|\tau_{n}\right\|_{n}^{2} \leqslant\|\tau\|_{n}^{2}$ $\left(\tau \in K_{n}\right)$.

THÉorÈme.

$$
\begin{gathered}
\tau_{n} \rightarrow \eta \quad(n \rightarrow \infty), \\
\left\{\left\|\tau_{n}\right\|_{n}^{2}-\|\eta\|^{2} \mid \leqslant c_{1} n^{-1} \log n \quad(n \geqslant N),\right. \\
{\left[\tau_{n}-\eta\right] \leqslant c_{2} n^{-1 / 2} \log n \quad\left(n \geqslant N^{\prime}\right)}
\end{gathered}
$$

où pour une mesure $\sigma$ quelconque portée par $C$

$$
[\sigma] \text { df } \sup |\sigma(L)| \text {, }
$$

la borne supérieure étant prise pour tous les arcs de Jordan $L \subset C$ [5]; par suite, pour les branches convenables des logarithmes

$$
\left|\log \varphi_{n}(z) / f(z)\right| \leqslant c_{3} V(z) n^{-1 / 2} \log n \quad\left(n \geqslant N^{\prime}\right),
$$

où

$$
\begin{gathered}
\log 1 / \varphi_{n}(z) \stackrel{\text { d? }}{=} \sum_{i=1}^{n} m_{i n} \log \left(z-z_{i n}\right)^{-1}, \quad m_{i n}=\tau_{n}\left(\tilde{i}_{i n}\right) \\
V(z)=\underset{\zeta \in C}{\operatorname{Var} \log (z-\zeta)^{-1} .}
\end{gathered}
$$

Les nombres $N, N^{\prime}, c_{1}>0, c_{2}>0, c_{3}>0$ dépendent de $C$ seulement.

Nous obtenons alors les approximations $\varphi_{n}(\tilde{z})$ de la représentation conforme, dont la convergence est du même degré que celle des fonctions $f_{n}$ de M. Leja (8) (voir [14]). Pour calculer $\phi_{n}$ il faut trouver les nombres $m_{\text {in }} \epsilon\langle 0, x d \theta / n\rangle$ qui donnent la valeur minime à la forme

$$
\left\|\tau_{n}\right\|_{n}^{2}=\sum_{i \neq k} m_{i n} m_{k n} \log _{n}\left|z_{i n}-z_{k n}\right|^{-1}
$$

dont les coefficients sont choisis par nous-mêmes, sous la condition peu restrictive (9). L'estimation (13) et (14) résultant de (12), une erreur, 
par exemple, de $n^{-1} \log n / \vartheta$ dans $\left\|\tau_{n}\right\|_{n}^{2}$ est bien admissible - ce qui fait ressortir les exigences modérées de notre méthode quant à l'exactitude des calculs. La machine peut alors chercher les valeurs pour $m_{\text {in }}$ parmi les nombres $0,1 / n^{2}, 2 / n^{2}, \ldots, x d \theta / n$.

6. Démonstration du théorème. Soit $C_{i n}^{*} \subset C$ un arc de centre $z_{i n}$ et de longuenr $\left|C_{i n}\right| / n$, et $a$ une mesure auxiliaire sur $\bigcup_{i} C_{i n}^{*}$ définie par $a=\sum_{i} m_{i n} \eta_{i n}^{*}, \eta_{i n}^{*}$ désignant la mesure d'équilibre sur $C_{i n}^{*}\left(\mathrm{n}^{0} 2\right)$. Comme $\eta$ est minimale $\left(\mathrm{L}^{0} 2\right)$,

$$
\|\eta\|^{2} \leqslant\|a\|^{2}=\sum_{i, k} m_{i n} m_{k n}\left(\eta_{i n}^{*}, \eta_{k n}^{*}\right) .
$$

Cherchons à estimer cette somme.

Le diamètre transfini $d\left(C_{i n}^{*}\right) \geqslant 1$ (diamètre de $\left.C_{i n}^{*}\right) \geqslant c_{4} n^{-2}$ (par example [9]) done par (3) on a $\left(\eta_{i n}^{*}, \eta_{i n}^{*}\right)=\log 1 / d\left(C_{i n}^{*}\right) \leqslant \log 1 / c_{4} n^{-2}$. Nous disons que, pour $i \neq k$ et $n$ suffisamment grand,

$$
\begin{aligned}
& \left|\left(\eta_{i n}^{*}, \eta_{k n}^{*}\right)-\log \right| z_{i n}-\left.z_{k n}\right|^{-1} \mid \\
& \quad \leqslant \iint_{c_{i n}} \int_{c_{k n}}|\log | \tilde{\sim}-\left.\zeta\right|^{-1}-\log \left|z_{i n}-z_{k n}\right|^{-1} \mid d \eta_{i n}^{*} d \eta_{k n}^{*} \leqslant c / n .
\end{aligned}
$$

En effet, par le théorème de la moyenne de Lagrange, $\left|\log a^{-1}-\log b^{-1}\right|$ $=|a-b| \cdot 1 / r$ pour un certain $r>\min \{a, b\}$. Posons $b=\left|z_{\text {in }}-z_{k n}\right|, a=|z-\zeta|$ $\left(z \in C_{k n}^{*}, \zeta \in C_{k u}^{*}, i \neq k\right)$, alors par (9) $r>v^{2} n^{-1}-2 \theta n^{-2}$ et $|a-b| \leqslant 2 \theta n^{-2}$, d'où notre inégalité. Done par (10)

$$
\begin{aligned}
\|\alpha\|^{2} & \leqslant \sum_{i} m_{i n}^{2} \log n^{2} / c_{4}+\sum_{i \neq k} m_{i n} m_{k n}\left(\log \left|\tilde{z}_{i n}-z_{k n}\right|^{-1}+c_{5} / n\right) \\
& \leqslant(x d \theta)^{2} n^{-1} \log n^{2} / c_{4}+\left\|\tau_{n}\right\|_{0}^{2}+c_{5} / n .
\end{aligned}
$$

Intruduisous une mesure de comparaison $\beta$, obtenue par la ,condensation" de $\eta$ sur $\left\{z_{i n}\right\}: \not \beta \in K_{n}, \not \beta\left(\left\{z_{i n}\right\}\right)=\eta\left(C_{i n}\right)$. Dans [6] nous avons démontré un lemme, dont la teneur s'exprime dans les présentes circonstances par l'inégalité

$$
\|\beta\|_{0}^{2} \leqslant\|\eta\|^{2}+c n^{-1} \log n .
$$

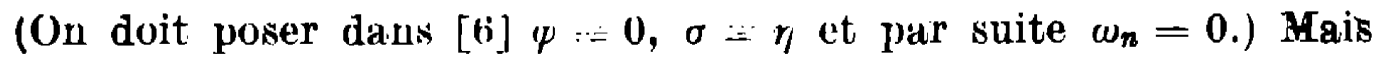

$$
\|\beta\|_{0}^{2}+\sum_{i} \eta\left(C_{i n}\right)^{2} \log n / \theta=\|\beta\|_{n}^{2}
$$


et $\sum \eta\left(C_{i n}^{*}\right)^{2} \leqslant(x d \theta)^{2} / n$, donc, par la définition de $\tau_{n}$,

$$
\left\|\tau_{n}\right\|_{n}^{2} \leqslant\|\beta\|_{n}^{2} \leqslant\|\eta\|^{2}+c_{7} n^{-1} \log n .
$$

Les inégalités (17)-(20) nous donnent (12) (voir (6), (7)).

Mais $U^{\eta}=\|\eta\|^{2}$ sur $C$, donc pour $\sigma=a-\eta$ :

$$
\|\sigma\|^{2}=\|a\|^{2}-2(\eta, \alpha)+\|\eta\|^{2}=\|a\|^{2}-\|\eta\|^{2} \leqslant c n^{-1} \log u
$$

(aussi par (17)-(20)). Pour les densités $\alpha^{\prime}, \eta^{\prime}$ on a $\left|\alpha^{\prime}-\eta^{\prime}\right| \leqslant c n$ (car $\eta^{\prime}$ est bornée). Or, nous avons démontré des théorèmes concernant l'estimation de $[\sigma]$ par $\|\sigma\|$ à condition que la densité soit bornée. Nolamment, l'inégalité (31) de [5] nous donne, en vertu de (21),

$$
\frac{c_{8} \frac{\log n}{n}}{[\sigma]^{2}} \geqslant \frac{\|\sigma\|^{2}}{[\sigma]^{2}} \geqslant \frac{c}{\log \frac{c_{0} n}{M[\sigma]}} \quad(c>0) .
$$

Par des calculs élémentaires nous en déduisons

$$
[\sigma]=[\alpha-\eta] \leqslant c_{10} n^{-1 / 2} \log n,
$$

et comme $\left[\alpha-\tau_{n}\right] \leqslant \varkappa d \theta / n$, nous obtenons l'inégalité (13) qui entraîne (11) par la voie indiquée par le théorème VII de [5] (cf. théorème II daus [:]). Notre théorème est ainsi démontré.

Remarque 1. Une erreur des $z_{i n}$ qui ne dépasse pas $c_{11} / n^{2}$ cause un changement de $\left\|\tau_{n}\right\|_{n}^{2}$ de $e_{12} / n$ au plus. Dans les caleuls numériques on peut donc calculer les coordonnées des $z_{i n}$ à $c_{11} / n^{2}$ près. La valeur de cin peut être choisie arbitrairement quant à la convergence de la méthode, mais l'estimation (12) augmente approximativement de $c_{11} / n \vartheta$, et celles de (13) et (14) subissent des changements correspondants.

Remarque '2. Le lecteur a sans doute observé que toutes nos considérations peuvent être menées à bonne fin avec la forme \|\|$_{j}^{2}$ au lieu de \|\|$_{n}^{2}$. Nous avons introduit cette seconde forme à cause des généralisations dont notre méthode semble capable et qui seraient impossibles avec la première.

\section{Travaux cités}

[1] N. Bourbaki, Intégration (Él. de math., L. VI chap. III) At. Sci. Ind. 1175, Paris 1952.

[2] M. liekete, Über die Verteilung der Wurzeln bei gewissen algebratsohe'n Glet. ehungen mit ganzzahligen Koeffizienten, Math. Zoitsch. 17 (1932), pp. 228-249.

[3] O. l'rostman, Yotentiel d'équilibre, Lund 1935.

[4] W. Kleiner, Sur l'approximalion de la représentation conforme par la methode des poinls extrémaux de M. Leja, Ann. Pol. Math. I4 (1963), [p. 131-140. 
[5] - Une condition de Dini-Lipsohitz dans la théorie du potentiel, Ann. Pol. Math. 14 (1963), pp. 117-130.

[6] - Sur la condensation des masses, Ann. Pol. Math. 15 (1964), pp. 85-80.

[7] М. А. Лаврентьев, Б. В. Шабат, Методы теории функиии комплексного переменного, Москва-Ленинград 1951.

[8] F. Leja, Sur une suite de polynômes et la représentation conforme d'un domaine plan quelconque sur le cercle, Ann. Soc. Pol. Math. 14 (1935), pp. 116-134.

[9] - Teoria /unkeji analitycznych, Warszawa 1957, str. 558.

[10] G. Pólya, G. Szegö, Über den trans/initen Durchmesser (Kapazitatskon. slunte) von ebener und räumlichen Punktmengen, Jouru. f. Math. 165 (1931), pp. 4.49.

UNIVERSITE JAGELLONNE

INSTITUT MATHEMATIQUE DE LACADEMIE POLONAISE DES SCIENCES

Keşu par la Rédaction le 20.9. 1962 